\title{
Increasing choice of and access to family planning services via outreach in Rajasthan, India
}

\author{
Kumudha Aruldas \\ Population Council \\ M.E. Khan \\ Population Council \\ Jaleel Ahmad \\ Population Council \\ Anvita Dixit \\ Population Council
}

Follow this and additional works at: https://knowledgecommons.popcouncil.org/departments_sbsr-rh

Part of the Demography, Population, and Ecology Commons, Family, Life Course, and Society

Commons, International Public Health Commons, Maternal and Child Health Commons, and the Women's Health Commons

How does access to this work benefit you? Let us know!

\section{Recommended Citation}

Aruldas, Kumudha, M.E. Khan, Jaleel Ahmad, and Anvita Dixit. 2014. "Increasing choice of and access to family planning services via outreach in Rajasthan, India." New Delhi: Population Council. 
Increasing choice of and access to family planning services via outreach in Rajasthan, India

An evaluation of Marie Stopes India's outreach services Population Council May 2014

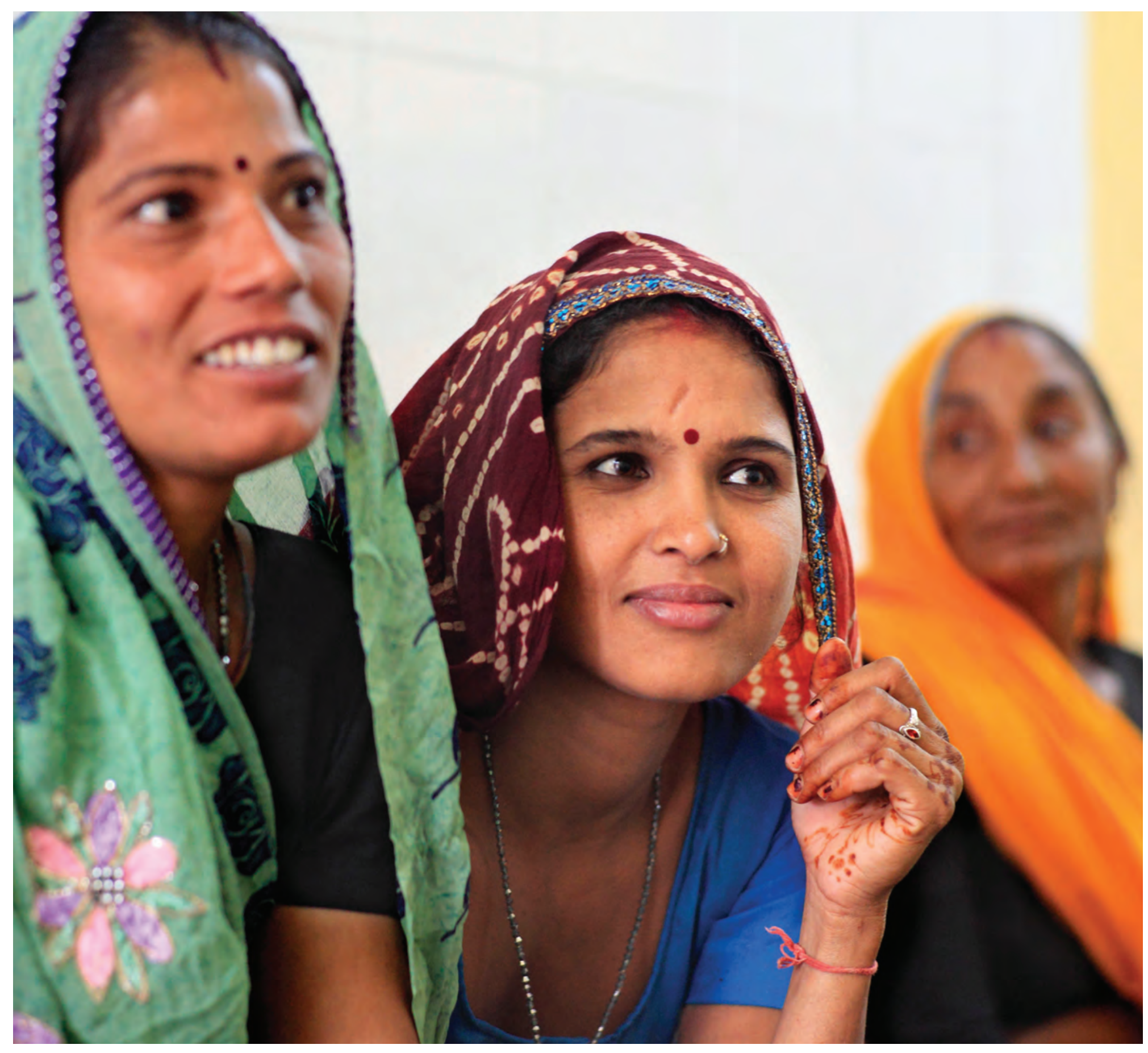




\section{Acronyms}

ASHA Accredited Social Health Activist

ANM Auxiliary Nurse and Midwife

AWW Anganwadi Worker

CHC Community Health Center

CHW Community Health Worker

COT Clinical outreach teams

CPR Contraceptive Prevalence Rate

FP Family Planningsciences

GOI Government of India

IPC Interpersonal Communicator

IUD Intra Uterine Contraceptive Device

LAPM Long-acting and permanent method
MSI Marie Stopes International

NPP National Population Policy

PHC Primary Health Center

OBC Other Backward Classes

SIFPO Supporting International Family Planning

Organizations

SLI Standard of Living Index

SRH Sexual and reproductive health

SPSS Statistical Package for the Social

Sciences

TL Tubal Ligation

USAID United States Agency for International Development

The Population Council confronts critical health and developmental issues, from stopping the spread of HIV to improving reproductive health and ensuring that young people lead full and productive lives. Through biomedical, social science, and public health research in 50 countries, we work with our partners to deliver solutions that lead to more effective policies, programs, and technologies that improve lives around the world. Established In 1952 and headquartered in New York, the Council is a nongovernmental, non-profit organization governed by an international board of trustees.

www.popcouncil.org

This study is made possible by the USAID Support for International Family Planning Organizations (SIFPO) project. SIFPO is implemented by MSI in partnership with EngenderHealth, the International Center for Research on Women, the International HIVIAIDS Alliance, and Population Council, aims to increase the use and availability of voluntary family planning services globally through strengthening MSI's capacity. SIFPO is a five-year program funded by the USAID under Cooperative Agreement No. AIDOAA-A-10-00059. The contents are the responsibility of the Population Council and do not necessarily reflect the views of USAID or the United States Government.

\section{Acknowledgements}

The Population Council would like to thank Monisha Vaid, Research and Evaluation Manager along with the MSI Rajasthan team for helping to coordinate with the Population Council's research team, Aziz Rahman for data entry and Louise Bury for assisting with the report edit.

\section{Suggested citation}

Aruldas, K., Khan, M. E., Ahmad, J., \& Dixit, A., (2014). Increasing choice and access to family planning services via outreach in Rajasthan, India: An evaluation of MSI India's outreach services New Delhi, India: Population Council.

\section{(C) 2013 The Population Council, Inc}

\section{Cover photograph credit}

Marie Stopes International

(c) Marie Stopes International 


\section{Contents}

1. Executive summary iii

2. Introduction 01

2.1 Family planning method mix in India 01

2.2 Access to family planning services 02

2.3 Marie Stopes India 02

$\begin{array}{ll}2.4 \text { Study aims and objectives } & 04\end{array}$

3. Methodology 05

3.1 Study design, location and participants 05

4. Findings 07

$\begin{array}{ll}4.1 \text { Study recruitment } & 07\end{array}$

4.2 Socio demographic characteristics $\quad 07$

4.3 Knowledge and source of information about contraception 10

4.4 Previous contraceptive use
4.5 Source 10

4.5 Source of information about outreach services and decision making 10

4.6 Side effects and incidence of complications 11

4.7 Follow up care and health seeking behaviour by day 15

4.8 IUD discontinuation by day 90 post procedure 15

$\begin{array}{lr}4.9 \text { Client Satisfaction } & 17\end{array}$

5. Discussion and recommendations 18

$\begin{array}{ll}5.1 \text { Conclusions } & 20\end{array}$

5.2 Recommendations $\quad 20$

5.3 Evidence to Action: Marie Stopes India response to recommendations 21

6. References 22

List of tables

Table 1: Family planning data for Rajasthan State and India 01

Table 2: Severity level of side effects 06

Table 3: Distribution of TL and IUD clients recruited for the study by district 08

Table 4: Client socio-demographic characteristics for TL and IUD services 09

Table 5: Types of side effects and severity reported by clients post procedure 12

Table 6: Types of side effects / complications and severity reported by TL clients

Table 7: Types of side effects / complications and severity reported by IUD clients

15 days post procedure $(n=369) \quad 14$

Table 8: Health seeking behaviour among women up to day 15 following TL and IUD procedure 15

List of figures

Figure 1: Study cohort profile 08

Figure 2: Reasons cited for choosing MSI outreach services IUD / TL (\% of women) 10

Figure 3: Reasons cited by clients for adopting tubal ligation and IUD -

multiple answers given (\% of women) 11

Figure 4: Flow chart showing IUD discontinuation by day 90 among IUD study cohort 16

Figure 5: Self reporting of continued side effects of IUD at day 90 (\% of women) (n=393) 16 


\section{Executive summary}

This report documents a service evaluation of Marie Stopes (MS) India's mobile outreach programme in the state of Rajasthan, which was conducted by the Population Council in India between March and October 2012. This study was made possible through the United States Agency for International

Development (USAID) funded Support for International Family Planning Organizations - Marie Stopes International (SIFPO-MSI) project. It provides important findings on the safety and effectiveness of MS India's outreach programme in expanding choice and access to long-acting reversible contraception (LARC) methods and voluntary surgical permanent methods (PM) of contraception, as well as recommendations for MS India and other family planning programmes to consider in scaling up and strengthening provision of mobile outreach services.

\section{Family planning in India}

India has a modern contraceptive prevalence rate of $48 \%$ [2]. One in five married women has an unmet need for family planning; $13 \%$ of married women have an unmet need for contraception to limit childbearing, while $7 \%$ of married women have an unmet need for spacing [12]. Unmet need is more acute in rural settings where health services and personnel are lacking. To address this, in recent decades the Government of India (GOI) has focused on expanding access to voluntary Family planning services. This was most recently articulated in the National Population Policy (NPP 2000), which emphasises the need to continue to provide reproductive healthcare and family planning services using a voluntary, informed consent and target-free approach. NPP 2000 has been bolstered by $\mathrm{GOI}$ strategies aimed at reducing the cost burden of family planning services, in particular costs and barriers associated with higher-cost permanent methods.

The family planning method mix in India remains dominated by voluntary permanent methods. Currently, nearly three quarters (71\%) of modern method family planning users in India have opted for female sterilisation. Despite high levels of effectiveness and user satisfaction [3], LARC uptake is very low, with only $2 \%$ of women using the intrauterine device (IUD). The contraceptive implant is not yet registered in India; therefore, use of LARC is limited to IUDs. A lack of access to and uptake of LARC stems from weak political support, prevailing misconceptions amongst potential users, and poor provider counselling. Contraceptive injectables are available in private sector facilities but are not offered in the public sector where short-acting methods are limited to oral contraceptive pills and condoms. Emergency contraception is also available. In India, uptake of condoms stands at $6 \%$ and uptake of pills at $4 \%$ [12]. Permanent methods for males (vasectomy) represent approximately $1 \%$ of modern method use [12].

Despite a decline in the country's total fertility rate (TFR), this has not translated to improved maternal and infant health, partly due to early marriage and short intervals between births. In order to improve women's health and enable couples to achieve desired family size, it is crucial to improve current low contraceptive access and limited choice among family planning methods, particularly in rural and poor regions of India.

\section{Marie Stopes India}

Marie Stopes India was established in 2008 to work in partnership with the GOI health departments to provide reproductive health and family planning services to poor men and women living in rural and remote areas. Partnership with non-governmental service providers is a key strategic approach for $\mathrm{GOI}$ implementation of frameworks such as the National Population Policy (NPP 2000).

Although the GOI offers family planning services through public sector facilities and has been active delivering permanent methods through outreach, GOI capacity is limited and, therefore, significant demand for PMs and LARC in rural districts remains unmet. The private (commercial) health sector in India is active, yet scarcity of private clinics in rural regions somewhat limits the private sector's role in service delivery to the hardest to reach and poorest clients. Through collaboration with the GOI to provide outreach services, MS India fills gaps in existing service availability. These gaps, which MS India address through mobile outreach, centre on access to IUDs, tubal ligations (TL), and vasectomy. Contraceptive 
implants are not yet registered in India, and injectables are not offered in public sector facilities. Condoms, oral contraceptive pills, and emergency contraception are available in public sector facilities (alongside MS India provision of PMs and LARC). During government stock-outs, MS India supports the public sector by supplementing their stock of short-acting methods.

MS India currently works across Rajasthan, Uttar Pradesh and Madhya Pradesh states. In 2011, MS India provided a total of 109,074 TLs and 34,091 IUD procedures, which contributed to $32 \%$ of the total TLs and $8 \%$ of all IUDs provided in the state of Rajasthan in 2011 . [4] Data from client exit interviews demonstrates that on average, $80 \%$ of MS India's outreach clients were not using a modern family planning method before taking up a service through outreach.

\section{Study Objectives and Design}

While expanding the availability of family planning methods is important, it is equally important that the quality of these services is maintained to ensure that family planning procedures are safe and meet the expectations of clients in outreach settings. Under the auspices of the USAID-funded SIFPO-MSI project, Population Council carried out an evaluation of MS India's mobile outreach services to provide a better understanding of the safety, acceptability, and profiles of clients accessing contraceptive services through MSI's outreach service delivery in rural areas. Findings from the study will inform and improve further family planning outreach programming.

The study focused on TL and IUD procedures provided by MS India in four districts of Rajasthan. These methods were chosen as they represent the vast majority of MS India's contribution to the method mix of family planning services offered at outreach sites. Vasectomy services were excluded from the study due to sporadic client numbers.

Between March and October 2012, 875 women receiving a TL and 402 women receiving an IUD procedure via MS India outreach were recruited for the study. Clients were interviewed before and immediately after their procedure at the outreach site, and on day 15 post procedure, about their side effects and satisfaction. Women who received an IUD were also interviewed at day 90 where information was collected about method discontinuation and reasons for IUD removal.

\section{Study Findings and Recommendations}

The study demonstrates that TL and IUD procedures can be provided safely and effectively in rural and hard to reach areas of Rajasthan through MS India's mobile outreach approach. In the selected cohort no major complications were reported and side effects were generally mild or moderate. The incidence of side effects and complications observed in this study are largely in line with published literature.[1,5-10] Most of the clients were willing to recommend the service to other women, signalling strong client satisfaction. The majority of women were from rural areas (81\% of TL clients; $72 \%$ of IUD clients), poor households and had no education (53\% of TL clients; $30 \%$ of IUD clients), which shows that through this service MS India is also fulfilling its objective in reaching underserved women in rural settings. Before taking up a family planning method from MS India mobile outreach, $63 \%$ of TL clients and $41 \%$ of IUD clients had never used modern contraception.

These findings support the recommendation that, in India, family planning programmes should consider mobile outreach models to broaden the range of services available in rural and hard to reach areas. Through mobile outreach, MS India is able to add LARC and PMs to the method mix available in government facilities, complementing the governmentprovided short-acting methods to offer clients a wider choice. In addition to service provision, MS India should continue to advocate for the registration of implants, wider availability of injectables, and promote access to a broader method mix in India. 


\section{Introduction and background}

\subsection{Family planning method mix in India}

There is a large unmet need for voluntary family planning in India, with an estimated 32 million or $13 \%$ of currently married women who want to limit their children and a further $7 \%$ who would like to space the time interval between multiple births, but who are not using any modern method of contraception. [12] Over recent decades, the country has seen a focus, largely driven by the GOI, on expanding access to voluntary family planning methods including permanent methods (PMs).

Unique to India, the adoption of female sterilization, which has steadily increased over the past two decades, tends to occur at a relatively young age following early marriage and childbearing, [13] and after the birth of at least one son. [2] More than half of women who choose a TL have the procedure before they reach 26 years of age, and in some states the average is as young as 23 years. [2]
In Rajasthan and more broadly in India, TLs represent a significant proportion of the contraceptive method mix (see Table 1). Low uptake of LARCs is due to multiple factors including weak political commitment for these methods, registration barriers, myths and misconceptions, and poor provider counselling. LARCs, such as IUDs and implants have high levels of effectiveness and user satisfaction globally [3, 15] but in India only $2 \%$ of women use IUDs and none use implants (see Table 1). Globally, the IUD has the lowest discontinuation rate, compared to other shortand long-acting methods of contraception (a review of DHS data from 18 developing countries showed that the overall discontinuation rate of the IUD was $15.5 \%$ at 12 months following insertion). Low discontinuation can probably be credited to the IUD's low failure rate as well as the necessity to visit a health professional to have it removed. [16]

TABLE 1: Family planning data for Rajasthan State and India

\begin{tabular}{|c|c|c|}
\hline Indicator & Rajasthan & India \\
\hline Total fertility rate & 3.1 & 2.5 \\
\hline Contraceptive prevalence rate (CPR) for modern methods (\%) ${ }^{2}$ & 55.3 & 48.0 \\
\hline Female sterilisation & 42.6 & 35.8 \\
\hline IUD & 1.3 & 1.8 \\
\hline Male sterilisation & 0.6 & 1.1 \\
\hline Pill & 2.7 & 3.6 \\
\hline Injection ${ }^{3}$ & 0.2 & 0.1 \\
\hline Condom & 7.7 & 5.5 \\
\hline Traditional methods (withdrawal, periodic abstinence) & 2.7 & 6.4 \\
\hline Total unmet need for family planning & 16.9 & 20.5 \\
\hline Unmet need for spacing births & 7.0 & 7.2 \\
\hline Unmet need for limiting births & 9.9 & 13.3 \\
\hline Maternal mortality ratio $(\mathrm{MMR})^{4}$ & $\begin{array}{l}318 \text { per } 100,000 \text { live } \\
\text { births }\end{array}$ & $\begin{array}{l}212 \text { per } 100,000 \text { live } \\
\text { births }\end{array}$ \\
\hline
\end{tabular}

Source: International Institute for Population Sciences (IIPS). 2010. District Level Household Survey (DLHS-3), 2007-08: India. Mumbai. IIPS. Source: International Institute of Population Sciences (IIPS) and Macro International. 2008. National Family Health Survey (NFHS-3), 2005-06: Mumbai: IIPS and Macro International.

Source: RGI, 2011. Special bulletin on maternal mortality in India. SRS Bulletin. Office of Registrar General, Ministry of Home Affairs, India. Accessed from http://www.censusindia.gov.in/vital_statistics/SRS_Bulletins/Final-MMR\%20Bulletin-2007-09_070711.pdf 
LARC methods offer women the benefit of reduced risk of poor maternal and child health outcomes resulting from frequent and/ or many births [3] without women needing to rely on daily or per-sexualencounter action or on re-supply of contraceptive commodities. As reversible methods, LARC offer these benefits for women who wish to space or delay pregnancies. Offering LARC in the context of access to TL, vasectomy, and short-acting methods is critical to meet the differing needs and preferences of women and men, especially as needs may change over the span of life stages. Given the myriad of reasons for low uptake of LARC and relatively low uptake of shortacting methods in India, it is necessary to advocate and build capacity at multiple levels of the health system for a broader method mix and wider choice in contraceptive options.

\subsection{Access to family planning services}

Although the majority of users of modern contraception in India are TL adopters (71\% nationally and $77 \%$ in Rajasthan), access to a full range of family planning methods, including permanent methods, remains limited in under-served areas. Government health centres offer condoms and oral contraceptive pills but often lack providers with the clinical and counselling skills to effectively offer IUDs and do not have providers capable of delivering tubal ligation and vasectomy procedures. Injectables are not available in government facilities and contraceptive implants are not registered in India.

Private (commercial) sector facilities offer a wider range of method options, including condoms, pills, injectables, IUD and permanent methods. However, fees charged in the private sector can pose financial barriers for poor clients, and the scarcity of private facilities in rural areas limits the private (commercial) sector's ability to address unmet need in these regions.

To address the gaps in access for rural and underserved populations, the GOI engages in partnership with non-governmental service providers as a strategic approach to achieving key policy frameworks such as the National Population Policy (NPP 2000). Mobile outreach by MS India and other non-governmental service delivery organisations (e.g. FPAl) to provide family planning methods which government is unable to offer in rural areas represents an important strategy for filling gaps in method access. In Rajasthan, MS India is the only organisation providing mobile family planning services. The Family Planning Association of India (FPAI) implements mobile family planning outreach in other states.

\subsection{Marie Stopes India}

Marie Stopes India was established in 2008 to work in partnership with the GOI health departments to provide reproductive health and family planning services to poor men and women living in rural and remote areas of Rajasthan, Uttar Pradesh and Madhya Pradesh states. In Rajasthan, MS India provides outreach services in partnership with the government in 11 districts: Jaipur, Ajmer, Alwar, Nagaur, Sikar, Rajsamand, Bharatpur, Udaipur, Chittorgarh, Banswara, and Pratapgarh.

Delivering PMs and LARC, MS India complements the public sector's provision of short-acting methods offered at government facilities and at the community level by community health workers (ASHA, AWW, and ANM). In 2011-12, the number of services performed by MS India increased and the program has recently grown from six to 11 outreach teams covering 372 sites operating in eleven districts. In $201132 \%$ of the TLs and $8 \%$ of the IUDs provided in the state of Rajasthan were obtained from MS India.

MS India is well-positioned for sexual and reproductive health service delivery. As a member of the Marie Stopes International (MSI) global partnership, MS India promotes and adheres to MSI's global clinical protocols [21], training, monitoring, and standardised quality assurance mechanisms. MS India emphasises client focus, meaning that clinical quality, client trust and respect, and overall client experience are key. To ensure adherence to these standards, MS India regularly conducts peer assessments, internal clinical audits, and external clinical quality assurance using the MSI global monitoring framework, the Quality Technical Assistance (QTA). QTA uses a standardised tool to assess clinical quality compliance in service delivery covering counselling, service provision, infection prevention, management of medical emergencies, clinical governance, client focus, and management of equipment and supplies. For each annual QTA audit, a random sample of MS India providers from each 


\section{The Marie Stopes International (MSI) outreach model}

In order to increase access to family planning and broaden the contraceptive method mix available to underserved populations, MSI uses a mobile outreach model in over twenty countries to provide affordable, high quality family planning services, including LARC and PMs, to men and women living in rural and hard to reach areas. Sensitisation and information provision about the outreach services and the different contraceptive methods are conducted in advance of the outreach visits, often with the assistance from community health workers. Outreach teams work in coordination with local governments and utilise existing public health facilities (or tents where no facilities are available). The frequency, with which outreach teams return to a location, as well as the duration of each visit, depends on the level of demand. MSI mobile outreach programmes follow global clinical quality standards and implement rigorous follow up systems that allow women to access medical advice after their treatment.

service delivery area is assessed. Regular trainings for clinical providers ensure that skills are always up-todate.

MS India mobile outreach services are known as Clinical Outreach Teams (COT). These seven-member teams are comprised of three doctors - a surgeon, a medical officer, an anaesthetist (required by GOI) one male and one female nurse, one health care assistant, and one driver. In line with the GOI protocol only doctors are permitted to provide TLs whereas nurses or Auxiliary Nurse Midwives (ANMs) are authorized and trained to provide IUD services. The COTs provide services in government facilities such as primary health centres and community health centres, and men and women in the outreach catchment area are informed about the services by MS India's Interpersonal Communicators (IPCS) and government community health workers known as Accredited Social Health Activists (ASHAs).

Both IPCs and ASHAs are members of the community who create awareness of MS India's outreach services and provide information about family planning methods. The IPCs and ASHAs also work with the government ANMs to mobilise clients at the outreach site and to provide follow up care information. As a part of the MS India outreach service protocol all clients are informed about expected side effects, appropriate follow up care, check-up visits with the ANM and what to do if they experience any complications following their procedure. Both TL and IUD clients are also provided with a supply of analgesia medicine to take with them post procedure. All follow up care is carried out by the government ANM who visit clients as informed by the MS India's IPCs. If complications occur beyond the management capacity of ANMs and IPCs, clients are referred to higher level facilities or a MS India static clinic (depending on nature of complication and proximity). In 2011, MS India provided a total of 109,074 TLs, 34,091 IUD procedures amounting to $1,574,781$ CYP through its outreach programme. Clients choosing short-acting methods received their methods through the government facility at which outreach took place, and are therefore not represented in MS India's direct service provision client numbers.

MS India adheres to GOI and MSI global clinical protocols [21] for all services provided through mobile outreach (as well as through MS India's other service delivery channels). This includes comprehensive pre-procedure group family planning counselling on a broad range of contraceptive methods available in India. Clients receive individual counselling to discuss method preferences and provide detailed information on benefits and possible side effects of the chosen method. Client eligibility screening follows MSI clinical standards, i.e. pregnancy status, STI screening for IUD clients. [21] Clients who are eligible and wish to proceed with their LARC or PM sign informed consent forms in their local language (Hindi). For clients who cannot read or write, the consent form is explained in Hindi, and they thumb print their acceptance. TL, vasectomy and IUD clinical procedures follow GOI and MSI global clinical protocols. All clients receiving TL, vasectomy or IUD from MS India are counselled on managing post-procedure care and possible side effects. Clients are provided with analgesics to take home with them for pain management. Clients are given the mobile phone number of an MS India clinical staff member to contact in case of any complications or questions. 
For TL (and vasectomy) clients, MS India contacts them by telephone within 48 hours of their procedure. This contact is followed up by a visit by a government ANM on the third, fifth and 15th day after the procedure. All IUD clients receive three visits by a government ANM, timed to take place after their first post-procedure menstrual cycle, three months postprocedure, and 12 months post-procedure. No additional or different pre- or post-procedure clinical protocols were followed for the purposes of this study. Rather, participating clients were asked to return to the facility where they obtained their service 15 days post-procedure for TL clients and 15 days and 90 days post-procedure for IUD clients. These additional visits were non-clinical and exclusively for the purposes of data collection for this study (see section 3.1).

While expanding contraceptive access and choice is very important, it is equally important to maintain quality and to ensure that services are safe and meet the expectations of clients. MSI has a number of tools through which service quality is routinely monitored and client experiences and satisfaction is measured. To further inform and improve its family planning services, a more in-depth service evaluation among women receiving TL and IUDs procedures via MSI India's mobile outreach clinics in the state of Rajasthan was conducted and originally documented by the Population Council in collaboration with MS India for the Support for International Family Planning Organizations (SIFPO)-MSI project.[17, 18] The evaluation measured the prevalence of side effects and complications, health seeking behaviours, and discontinuation of IUDs, as well as the sociodemographic profile and satisfaction levels of clients receiving these mobile outreach services.

\subsection{Study aims and objectives}

The overall aim of this service evaluation was to provide more understanding about the safety, acceptability and profile of clients who access TL and IUD procedures via MS India's mobile outreach in rural areas. Through outreach in government facilities, MS India fills gaps in access to a LARC and PMs, helping ensure a broad contraceptive method mix is available to women and men in rural and hard-to-reach areas. With contraceptive implants unavailable in India (currently not registered) and a significant but irregular client volume for vasectomy, this study focuses on the provision of TL and IUD, methods that provide women with a choice of LARC and PM in rural India. This study's findings will help improve MS India's provision of services as well as inform other organisations currently active in outreach service delivery. Specific attention is paid to how service quality can be assured and key programmatic areas such as counselling messages and follow-up care mechanisms can be strengthened and replicated.

The evaluation has five objectives:

1. To measure the prevalence of side effects (and the severity of side effects) associated with TL and IUD procedures offered in MS India's mobile outreach sites

2. To assess the discontinuation rate at 90 days post procedure associated with IUD when delivered via outreach services

3. To explore clients' health seeking behaviours post procedure

4. To determine the client profile and proportion of "poor" clients who receive TL and IUD services via MSI India's outreach services; and

5. To evaluate clients' satisfaction with MSI's TL and IUD outreach services.

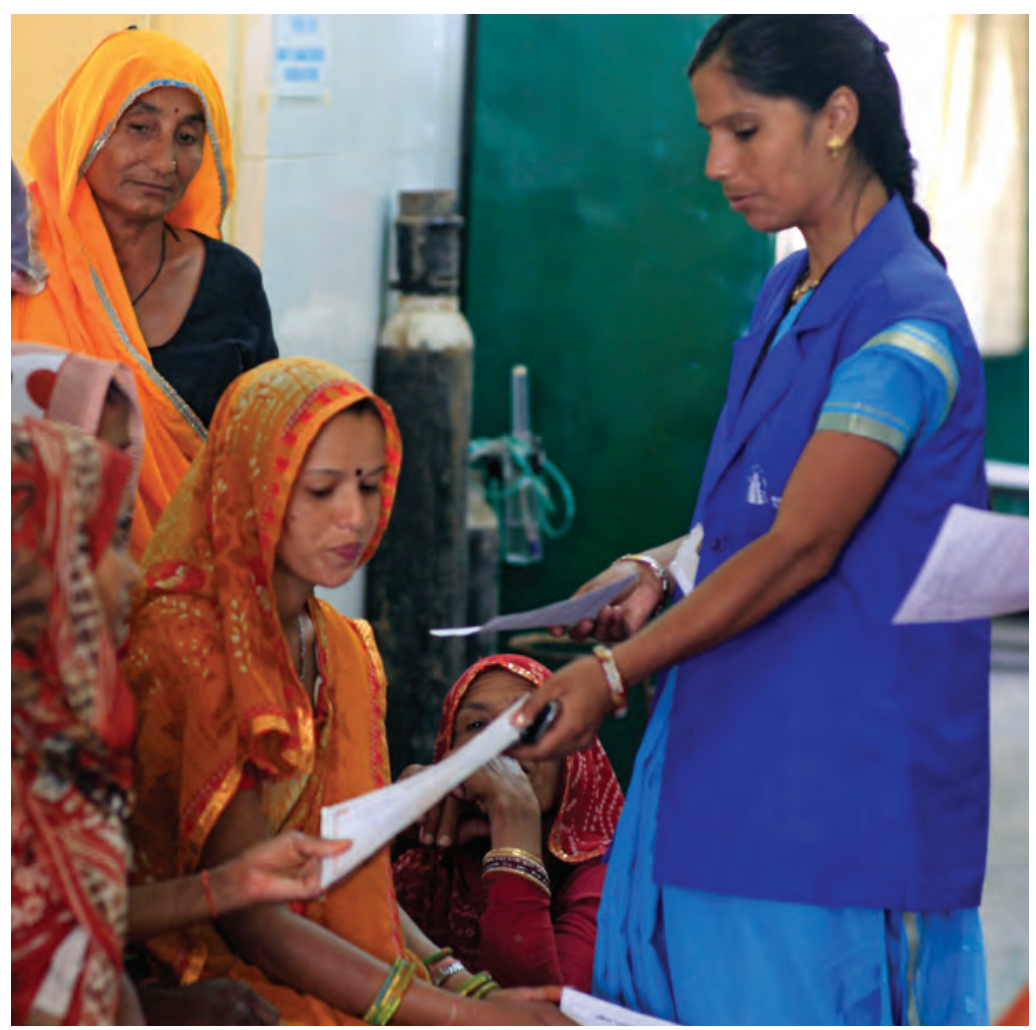




\section{Methodology}

\subsection{Study design, location and participants}

This evaluation involved following a cohort of women who received TL and IUD procedures from MS India between March and October 2012 at 235 mobile outreach sites. The sites were located in four districts in the state of Rajasthan (Jaipur, Ajmer, Alwar and Naguar) that were purposively selected on account of having the highest services numbers for these contraceptive services during 2010-2011.

Women were included in the study if they met the following eligible criteria: received TL or IUD via outreach services during the study period and were willing to give written or verbal informed consent to participate in the evaluation in their local language (Hindi). Written consent was provided in Hindi and verbal consent given (in Hindi) for the minority of clients who could not read or write.

The Population Council India office followed the IRB submission requirement. This study was exempted from full IRB review since it was not a clinical study; did not involve vulnerable populations; did not expose study subjects to more than minimal risks (e.g., health, legal, physical, social, economic, or psychological); did not involve voice or video recording.

\section{Sampling}

At the time of the study, a total of five outreach teams (Jaipur had two teams) were operating in the four districts included in the study and provided both TL and IUD services. The sample size was calculated separately for TL and IUD procedures to ensure that enough clients were recruited for each strata, and were divided between the 5 teams until the sample size was achieved.

The following formula was used for calculating the sample size, where $\mathrm{N}$ is the sample size required for the study and $z_{1-\alpha / 2}$ is the level of confidence taken as $95 \%$.

$N=\frac{z_{1-\alpha / 2}^{2} p(1-p)}{d^{2}} \times$ deff

For the TL client sample size, the baseline indicator $(p)$ was taken as 3\% which was based on findings from other studies that documented a range of 1-4\% complication rate for TL procedures carried out in similar settings $[7,8]$. A margin of error (d) of $2 \%$, a design effect (deff) of two was used to provide a sample size of 745 . In addition, a loss to follow up rate of $25 \%$ was then used to provide an overall sample of 800 TL clients.

For the IUD client sample size, the baseline indicator (p) for a discontinuation rate after 90 days of $15 \%$ was used based on an evaluation of MSI outreach services in other countries which documented discontinuation rates from $2.3-20.9 \%$.[9] A margin of error (d) of $1.5 \%$, a design effect (deff) of 1.5 provided a sample of 392. Considering a loss to follow up rate of $25 \%$ a total of 400 IUD clients was required.

\section{Survey instrument, data collection and data} analysis

A research team of two to three female investigators and a supervisor to recruitment participants and collect data were trained by Population Council. These individuals were chosen from the pool of investigators and supervisors available in the states who work with research organisations as and when they are required to collect data. For the purpose of this evaluation, investigators were chosen based on their experience with the Population Council and their availability during the required period.

Clients were recruited for the study prior to their procedure at the outreach site. Following informed consent, participating clients were interviewed about their background characteristics; knowledge and source of information about the outreach services; previous use of contraception; and reasons for choosing TL or IUD, and for opting to receive the service from MS India. After the procedure and post recovery at the site, clients were interviewed again to obtain feedback concerning satisfaction and any immediate side effects associated with the procedure. These interviews were conducted privately by investigators appointed by the Population Council. One of the roles of supervisors was to ensure privacy from other clients and from MS India providers and government staff. The post-procedure interview took no longer than 10-12 minutes so that clients could leave without undue delay.

Both TL and IUD clients were asked to return on day 15 post-procedure to the facility where they received 
TABLE 2: Severity level of side effects ${ }^{5}$

\begin{tabular}{|l|l|}
\hline Level 1: Very mild & $\begin{array}{l}\text { The client experiences low level of discomfort that does not require any medical } \\
\text { intervention }\end{array}$ \\
\hline Level 2: Mild & $\begin{array}{l}\text { The client experiences some level of discomfort that only requires resting or } \\
\text { minimum level of medical intervention such as taking pain relief from a medical } \\
\text { shop }\end{array}$ \\
\hline Level 3: Moderate & $\begin{array}{l}\text { The client experiences more frequent level of discomfort that requires attention } \\
\text { from medical personnel, such as a doctor }\end{array}$ \\
\hline Level 4: Severe & $\begin{array}{l}\text { The client experiences frequent level of discomfort that requires either further } \\
\text { medical check-up or hospitalization }\end{array}$ \\
\hline
\end{tabular}

MS India services for a follow up interview. The cost of travel and refreshments for this interview was met by the study. At this visit, the interviewer collected information about side effects and any complications, care seeking behaviour, resumption of normal activities and satisfaction. Women receiving an IUD were also asked to return on day 90 when information was collected about method discontinuation. If clients did not return to the site for a follow up interview, attempts were made to contact respondents by telephone if they had agreed. All women were asked in Hindi for consent to be interviewed at both day 15 and 90.

This service evaluation recorded any incident of complications during the procedure as indicated in the client records and then clients' experience of any side effects immediately after the procedure following their recovery period. Later, delayed side effects and complications were recorded on day 15 . Side effects and complications were categorised by type of symptom and severity. As the study was conducted at outreach locations medical personnel were not available to assess or validate the side effects at follow up interviews. Therefore, to ensure self-reporting of side effects was consistent and simple for respondents to recall, the level of severity was measured by the type of medical intervention required (if at all) to address the problem. The definitions of level of severity are shown in box 2 . Minor or moderate side effects were later differentiated from moderate or severe complications to distinguish between expected side effects and those that are not expected (and possibly related to the procedure or post-operative conditions of the woman).

The study design, development of survey tools and data collection was managed by the Population Council. Data was entered using the Census and Survey Processing System (CSPro 5.0.1). Descriptive statistical methods were conducted for analysis to provide using the Statistical Package for Social Sciences (SPSS 18.0). 


\section{Findings}

\subsection{Study recruitment}

The sample was recruited from women attending 235 MS India outreach service days based in a variety of available facilities in each district, such as primary health centres, community health centres and a MSI clinic based in Ajmer district. The majority of services were delivered in rural locations (81\% of TLs and $72 \%$ of IUDs) with the remaining delivered in urban and peri-urban areas (19\% of TLs and $28 \%$ of IUDs).

During the study period 1423 clients attended outreach services for TL and IUD procedures, and out of these women 1277 were eligible to participate in the study. Those who were not eligible either could not go ahead with the procedure due to medical reasons identified during the consultation, or decided not to go ahead with the procedure. Table 3 shows the distribution of the sample for each of the four districts. All clients agreed to take part and as shown in Figure 1, among the 1277 study participants 875 received TL, and 402 had IUDs inserted. At day 15, 803 (92\%) of women who had received a TL procedure and 369 (92\%) of IUD clients completed a follow up interview. Of these, 718 TL clients and 342 IUD clients returned to the health facility, and 85 TL clients and 27 IUD clients were contacted through a follow up telephone interview. Loss to follow up for both TL and IUD clients was 8\% (72 for TL and 33 IUD). At the 90 day followup for IUD clients, $98 \%(n=393)$ of the original enrolled clients attended a follow up visit, with only $2 \%$ loss to follow up (see Figure 1).

\subsection{Socio demographic characteristics}

The median age of clients was 26 years for TL clients and 25.5 years for IUD clients. All women were married and had one or more children (see Table 4). Two thirds of women adopting the TL method of contraception were between the ages of 25-29 years (67\%); whereas the age of women adopting the IUD was more evenly spread, with less than one half aged 25-29 years ( $47 \%$ ), and one third $20-24$ years (30\%), and one quarter over the age of 30 years (23\%). The majority of TL and IUD clients had two children ( $40 \%$ and $44 \%$ respectively) before adopting their method of contraception; followed by one third (36\%) of TL clients and one quarter (22\%) of IUD clients having three children.

The majority of clients were Hindu (TL: $94 \%$ and IUD: $85 \%$ ) and belonged to disadvantaged groups classified in India as Other Backward Classes ${ }^{6}$ (TL: 53\% and IUD: $62 \%)$. One quarter of both TL (29\%) and IUD (24\%) clients were from Schedule Caste / Schedule Tribe. A large proportion of women had never been to school or only had primary education (TL: $69 \%$ and IUD: 47\%); and considerably more women opting for IUD than TL had achieved secondary or higher education ( $53 \%$ and $31 \%$ respectively). The majority of women were housewives (TL: $75 \%$ and IUD 84\%), with more women having a TL than IUD being a farmer or agricultural labourer (19\% and $8 \%$ respectively).

To assess the socio-economic status and poverty levels of women the study adopted the Standard of Living Index (SLI) that is widely used in other national surveys of India and therefore the results are comparable to similar population based surveys for the state of Rajasthan 7 . This method is based on assigning a measure for the household's type of toilet facility, source of lighting and drinking water, type of fuel for cooking and ownership of durable goods. The scores are then added up to produce an overall index that ranges from a low, medium and high SLI score. The low SLI is categorised as "poor".

More than half of TL clients belonged to households categorised as either low (25\%) or middle income (32\%) SLI. By comparison, most IUD clients belonged to households with a high SLI (61\%) and reported a slightly higher average monthly household income - of Rs 8,516 RS (US\$155) - than TL clients at Rs 7,400 (US\$148).

\footnotetext{
Other Backward Classes is a collective term, used by the Government of India, for castes other than scheduled castes and scheduled tribes, which are higher on social hierarchy than SC/ST but educationally and socially disadvantaged.

Such as the National Family health Surveys (NFHS) and District Level Household Surveys (DLHS) published by the International Institute for Popula-
} tion Sciences and ORC Macro. 
TABLE 3: Distribution of TL and IUD clients recruited for the study by district

\begin{tabular}{lll}
\hline District & Tubal ligation clients \% (n) & IUD clients \%(n) \\
\hline Ajmer & $28.0(245)$ & $10.4(42)$ \\
\hline Alwar & $16.9(148)$ & $40.3(162)$ \\
Jaipur & $28.7(251)$ & $38.1(153)$ \\
Nagaur & $26.4(231)$ & $11.2(45)$ \\
Total & $100.0(875)$ & $100.0(402)$
\end{tabular}

\section{FIGURE 1: Study cohort profile}

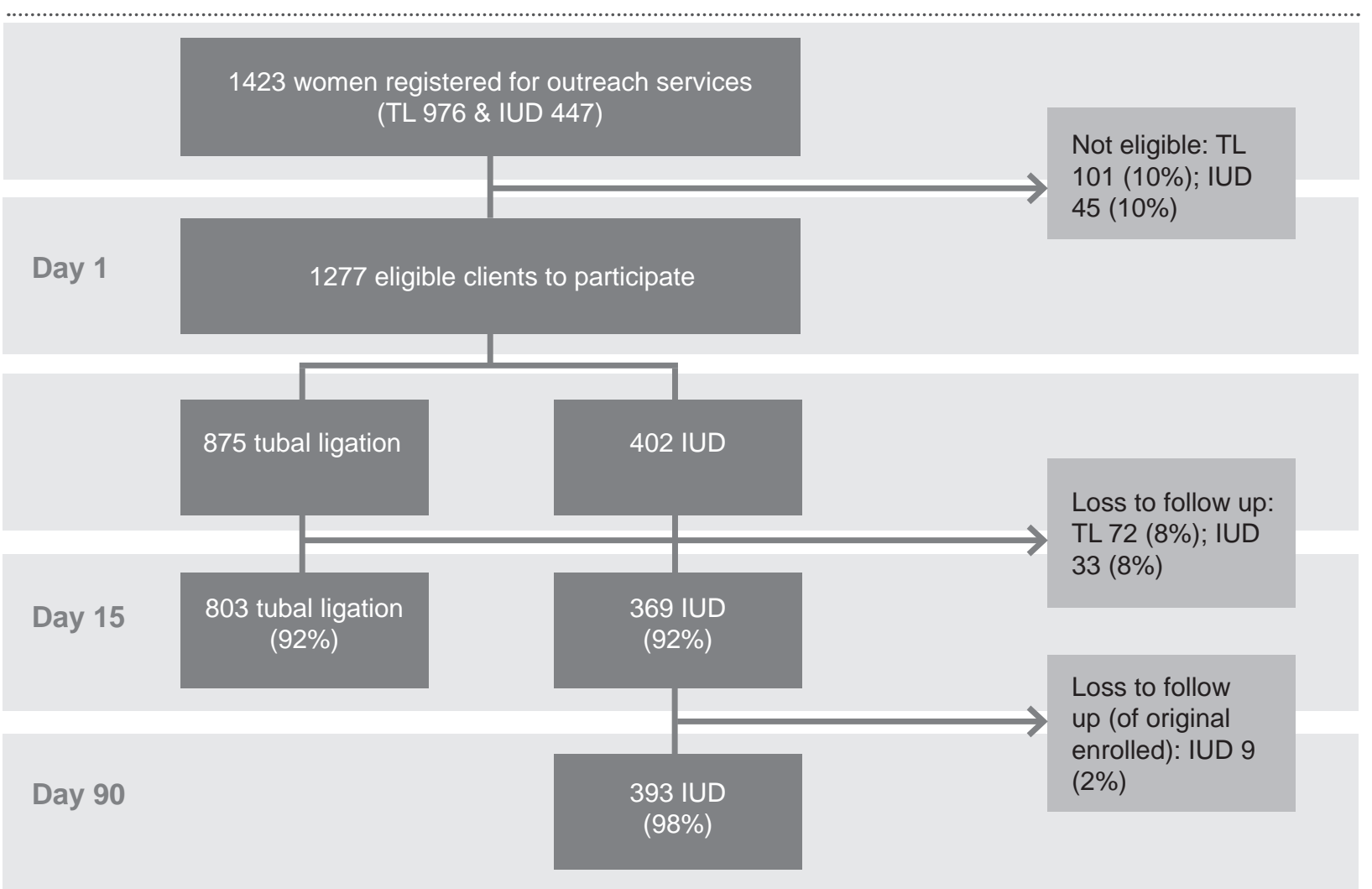


TABLE 4: Client socio-demographic characteristics for TL and IUD services

\begin{tabular}{|c|c|c|}
\hline Socio-demographic characteristics & $\begin{array}{l}\text { Tubal ligation clients } \\
(n=875) \%(n)\end{array}$ & $\begin{array}{l}\text { IUD clients } \\
(n=402) \%(n)\end{array}$ \\
\hline Age of women & 26.0 & 25.5 \\
\hline Under 20 (15-19) & - & $0.2(1)$ \\
\hline $20-24$ & $17.1(150)$ & $29.9(120)$ \\
\hline $25-29$ & $67.3(589)$ & $46.8(188)$ \\
\hline $30+$ & $15.5(136)$ & $23.1(93)$ \\
\hline Number of living children [mean] & 2.9 & 2.6 \\
\hline 1 & $0.5(5)$ & $15.2(61)$ \\
\hline 2 & $40.0(350)$ & $43.5(175)$ \\
\hline 3 & $36.2(317)$ & $22.1(89)$ \\
\hline 4 or more & $23.2(203)$ & $19.1(77)$ \\
\hline \multicolumn{3}{|l|}{ Religion } \\
\hline Hindu & $93.9(822)$ & $84.8(341)$ \\
\hline Muslim & $5.9(52)$ & $14.7(59)$ \\
\hline Other religious group & $0.1(1)$ & $0.2(2)$ \\
\hline \multicolumn{3}{|l|}{ Caste } \\
\hline Schedule caste/ Schedule tribe & $29.0(254)$ & $23.7(95)$ \\
\hline Other backward classes (OBC) & $53.1(465)$ & $61.7(248)$ \\
\hline General & $17.8(156)$ & $14.6(59)$ \\
\hline \multicolumn{3}{|l|}{ Education } \\
\hline Never attended school & $52.5(459)$ & $30.1(120)$ \\
\hline Primary education (class 1-5) & $16.8(147)$ & $16.9(69)$ \\
\hline Secondary education (class 6-9) & $22.4(196)$ & $36.3(146)$ \\
\hline Higher education (class 10 and above) & $8.3(73)$ & $16.7(67)$ \\
\hline \multicolumn{3}{|l|}{ Occupation } \\
\hline Housewife & $74.9(655)$ & $83.8(337)$ \\
\hline Farmer / agricultural laborer & $19.2(168)$ & $7.7(31)$ \\
\hline Other / petty business / laborer / community health worker etc. & $5.9(52)$ & $8.5(34)$ \\
\hline \multicolumn{3}{|l|}{ Standard of living index } \\
\hline Low & $24.7(216)$ & $14.2(57)$ \\
\hline Medium & $31.7(277)$ & $24.4(98)$ \\
\hline High & 43.7 (382) & $61.4(247)$ \\
\hline Average monthly household income & Rs. 7400 (US\$148) & Rs. 8515.71 (US\$ 155) \\
\hline
\end{tabular}




\subsection{Knowledge and source of information about contraception}

Knowledge about contraceptive methods was quite high among both TL and IUD clients, although the mean number of modern methods known was higher among IUD clients than for TL (4 and 2.8 respectively). Most commonly cited methods for TL clients were TL, condom and pill, with lower awareness of other LARCs and PM methods such as IUD, DMPA and vasectomy. Women who received an IUD expressed a wider knowledge of other contraceptive methods. The main sources of information about contraception for both TL and IUD clients were from Auxiliary Nurse and Midwives (ANMs), community based health workers (ASHA and Anganwadi workers [AWW]), husbands, and MSI IPC.

\subsection{Previous contraceptive use}

The majority of women were not using a contraceptive method before joining the study: $73 \%(n=639)$ of $T L$ and $54 \%(n=217)$ of IUD clients. Out of the $236 \mathrm{TL}$ clients who reported currently using a contraceptive method only 86 (36.4\%) could report how long they had been using the method. It is also unknown what type of family planning method (modern or traditional) was being used. Prior to adopting a LARC or PM at the MS India outreach service, 37\% $(n=325)$ of TL clients and $59 \%(n=236)$ of IUD clients reported having used contraception in the past. This finding implies that $63 \%$ of TL clients and $41 \%$ of IUD clients were new adopters of contraception.

\subsection{Source of information about outreach services and decision making}

The majority of women learnt about MS India outreach services from the facility based health workers or community health workers such as ASHA, AWW and ANM (TL 78\%; 56\% IUD). A lower proportion of women learnt about the outreach services from the MSI IPCs with $25 \%(n=217)$ of TL clients and $27 \%$, $(n=108)$ of IUD clients reporting that they had been contacted by the IPC in the community.

It is quite common for community health workers such as ASHAs to initially approach other family members when raising awareness about the outreach service as the decision to adopt family planning is strongly influenced by the husbands and parents-in-law. When there is agreement to consider family planning, women are then approached to discuss the services available.

FIGURE 2: Reasons cited for choosing MSI outreach services - IUD and TL (\% of women)

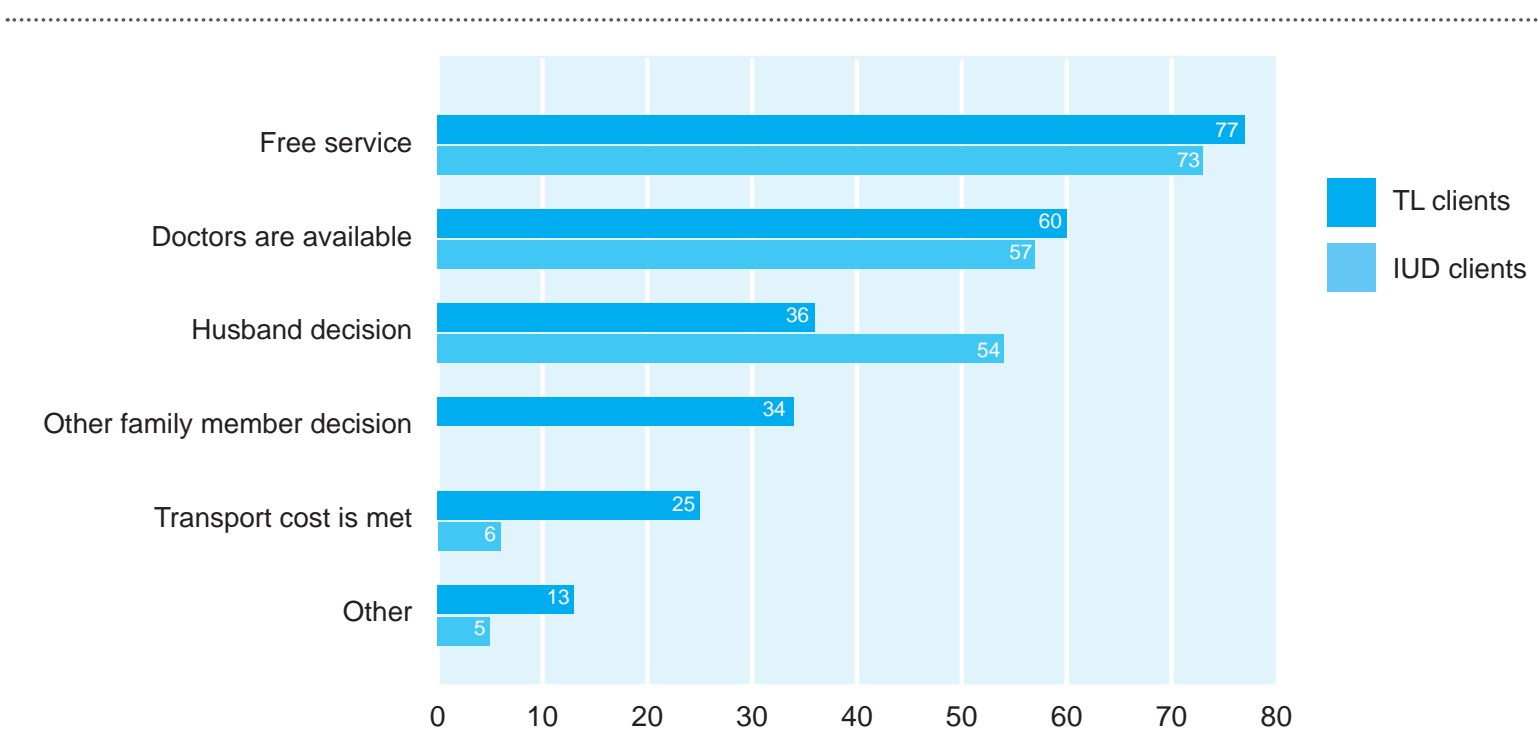


The main reasons cited by women for choosing the MSI India's outreach services were because the services were free $(77 \%, \mathrm{n}=673$ for $\mathrm{TL}$ and $73 \%$, $n=291$ for IUD), followed by the fact that clinical staff were available $(60 \%, n=521$ and $57 \%, n=228)$, (see Figure 2). More than half of IUD clients $(54 \%, n=217)$ compared to one third $(36 \%, n=315)$ of $T L$ clients said their husband's recommendation was the reason they used the service, and for women seeking a TL service one third also said they were recommended by another family member (34\%, n=293). Other reasons cited for choosing the outreach services were: financial reimbursement for travel costs, a better facility than alternative facility, proximity to home, and no other service available.

\section{Reasons for choosing TL and IUD methods}

There was much variation between the reasons cited by women for adopting TL and IUD methods of contraception (see Figure 3). Women were able to give multiple answers to why they chose their respective family planning method. The main reason for adopting TL was due to having reached a desired family size
(81\%, $n=707$ ) and for IUD the majority of women said their husband's preference for this method influenced their decision to use it $(68 \%, n=274)$. Influence of the husband or another family member on the woman's decision making was also commonly reported for reasons to adopt TL (48\%, $n=420$ and 38\%, n=335 respectively). Nearly half of IUD clients also said the influence of community health worker counselling and having reached their desired family size were why they chose the long acting method (45\%, $\mathrm{n}=183$ and 43\%, $n=173)$. One third of TL clients $(39 \%, n=341)$ also reported that they chose to be sterilised as their husbands did not agree to have a vasectomy.

\subsection{Side effects and incidence of complications}

Side effects on the day of the procedure were assessed from the clinical records to identify the types of side effects or complications that occurred during the procedure and post-procedural phase. Side effects on day 15 for both TL and IUD clients, and day 90 for IUD relied on the clients self-reporting of symptoms and level of severity.

FIGURE 3: Reasons cited by clients for adopting tubal ligation and IUD - multiple answers given (\% of women)

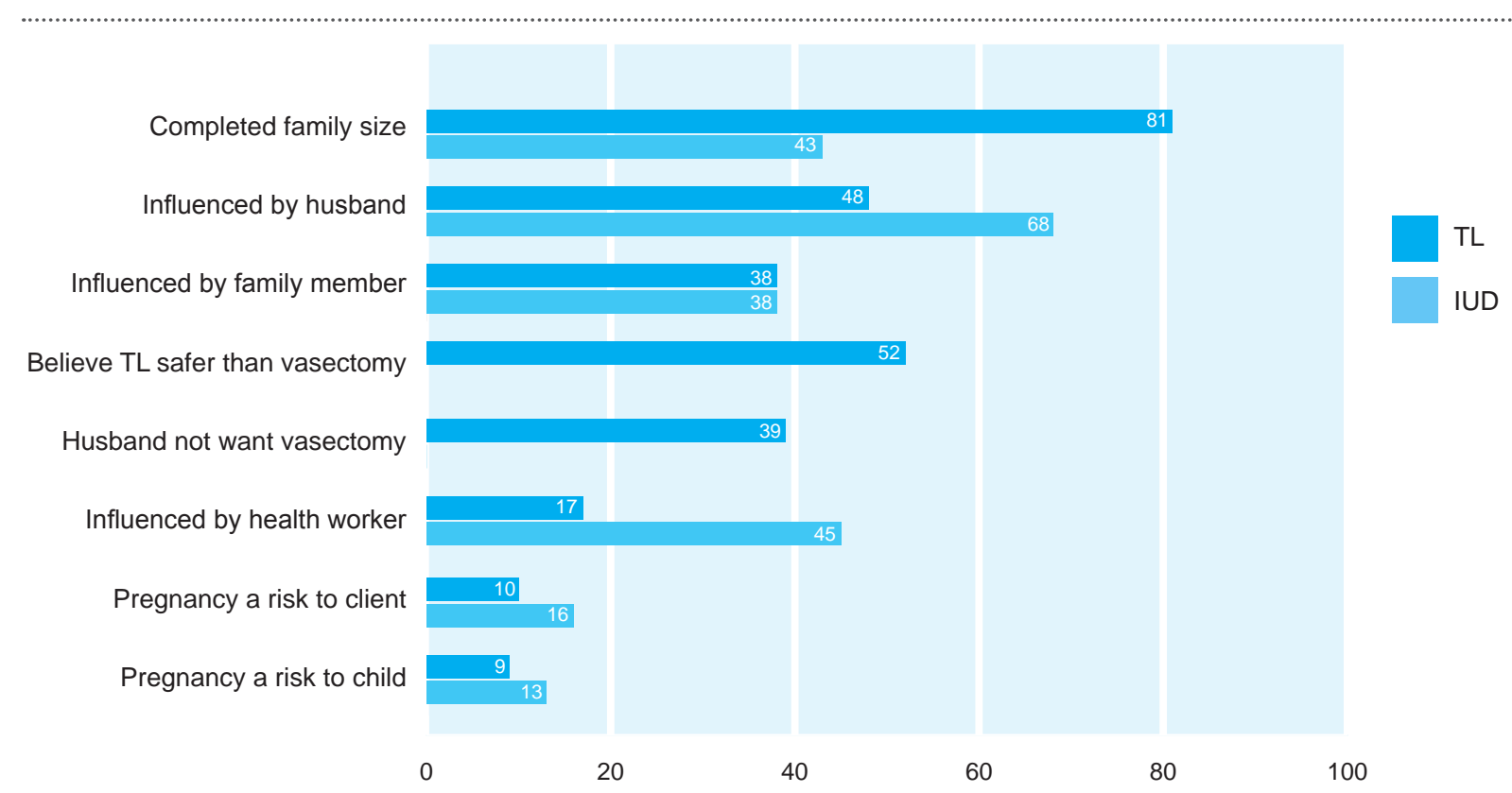




\section{Day of Procedure}

Information from clients' records showed that among 875 TL procedures, pain was recorded for $4 \%(n=32)$ at the time of the procedure. During the post operation observation period, pain was recorded for $10 \%(87)$, followed by vomiting $(0.3 \%, n=3)$ and fever $(0.1 \%, n=1)$ among TL clients. Among the 402 IUD procedures pain was recorded in $5 \%(n=20)$ of cases and dizziness $(0.5 \%, \mathrm{n}=2)$ at the time of insertion. No incident of complications related to both TL and IUD surgical operations at the time of the procedure or immediately afterwards were identified.
Following the procedure, 35\% $(n=308)$ of TL clients and $32 \%(n=94)$ of IUD clients reported experiencing side effects immediately after adopting their method (see Table 5). The majority of women experienced pain (34\% and 22\% for TL and IUD clients respectively), with $12 \%$ of TL clients reporting severe pain. One TL client also reported severe bleeding post procedure, but this case was managed by the outreach team and was not classified as a major incident.

TABLE 5: Types of side effects and severity reported by clients post procedure

\begin{tabular}{|c|c|c|c|}
\hline Side effects & & $\begin{array}{l}\text { Tubal ligation } \\
\text { clients } \\
\text { (n=875) } \\
\%(n)\end{array}$ & $\begin{array}{l}\text { IUD clients } \\
(n=402) \\
\%(n)\end{array}$ \\
\hline \multirow[t]{5}{*}{ Pain } & & $34.4(301)$ & $22.1(89)$ \\
\hline & Very mild & $14.7(129)$ & $16.6(67)$ \\
\hline & Mild & $16.2(142)$ & $4.2(17)$ \\
\hline & Moderate & $1.4(12)$ & $0.7(3)$ \\
\hline & Severe & $2.1(18)$ & $0.5(2)$ \\
\hline \multirow[t]{5}{*}{ Bleeding } & & $4.5(14)$ & $1.2(5)$ \\
\hline & Very mild & $1.3(11)$ & $0.4(2)$ \\
\hline & Mild & $0.2(2)$ & $0.7(3)$ \\
\hline & Moderate & - & - \\
\hline & Severe & $0.1(1)$ & - \\
\hline Other (vomiting, dizziness, weakness) & & $0.6(5)$ & - \\
\hline Total women reporting any side effects & & $35.5(308)$ & $32.4(94)$ \\
\hline
\end{tabular}




\section{Day 15 post procedure}

On day 15 , study participants were requested to return for an interview by the Population Council research team. This visit is separate from the routine follow-up and check-up by conducted by MS India and government providers that is carried out in the community (client's home). Information was obtained regarding the types of side effects women were experiencing at day 15 . Women could list multiple side effects. Among the women who had a TL who were interviewed $(n=803)$ 15\% $(n=119)$ reported at least one type of side effect, and for those who had an IUD $(n=369) 21.4 \%(n=79)$ said they were still experiencing some side effects.

Tubal ligation: As on the day of procedure pain was reported most frequently $(7 \%, \mathrm{n}=54)$ by $\mathrm{TL}$ clients, followed by abdominal pain $(6 \%, n=50)$ (see Table 4). Fewer women experienced problems with the wound $(1 \%, n=10)$, and less than $1 \%$ reported bleeding from the wound, urinary problems and a blood clot. At day 15 the majority of side effects were identified as minor with the majority able to seek medical care. A total of 31 women reported at least one moderate side effect (4\%) and one woman (0.1\%) had a serious complication and required hospitalization for one day due to bleeding from the wound and infection. Out of the 119 women who reported any side effect, the majority experienced very mild or mild levels of severity $(61 \%, n=72)$. Less than $4 \%(n=32)$ out of 803 TL clients reported a moderate or severe level of complication which required medical attention.

IUD: The most commonly reported side effects were pain $(11 \%, n=42)$ and bleeding $(11 \%, n=41)$ up to day 15 after IUD insertion (see Table 7). Five percent $(n=18)$ experienced abdominal swelling and / or pain, and less than one percent $(n=2)$ complained of having a urinary problem. One woman experiencing prolonged bleeding said that she was going to get the IUD removed. Compared to $\mathrm{TL}$, which involves a (small

TABLE 6: Types of side effects / complications and severity reported by TL clients 15 days post procedure ( $n=803$ )

\begin{tabular}{|c|c|c|c|c|c|}
\hline \multirow{2}{*}{$\begin{array}{l}\text { Side effect } I \\
\text { complication }\end{array}$} & \multicolumn{4}{|c|}{ Level of severity \%(n=803) } & \multirow{2}{*}{$\begin{array}{l}\text { Total } \\
\%(n)\end{array}$} \\
\hline & $\begin{array}{l}\text { Very mild } \\
\%(n)\end{array}$ & $\begin{array}{l}\text { Mild } \\
\%(n)\end{array}$ & $\begin{array}{l}\text { Moderate } \\
\%(\mathbf{n})\end{array}$ & $\begin{array}{l}\text { Severe } \\
\%(n)\end{array}$ & \\
\hline Pain in the wound & $2.8(23)$ & $1.0(7)$ & $3.0(24)$ & - & $6.7(54)$ \\
\hline Abdominal pain & $2.9(24)$ & 2.016 & $1.2(10)$ & - & $6.2(50)$ \\
\hline $\begin{array}{r}\text { Wound did not heal / } \\
\text { infection }\end{array}$ & $0.7(6)$ & $0.2(2)$ & $0.2(2)$ & - & $1.2(10)$ \\
\hline Wound oozing blood & $0.1(1)$ & $0.1(1)$ & $0.1(1)$ & $0.1(1)$ & $0.5(4)$ \\
\hline Urinary problem & $0.1(1)$ & - & $0.1(1)$ & - & $0.2(2)$ \\
\hline Blood clot & $0.1(1)$ & - & - & - & $0.1(1)$ \\
\hline $\begin{array}{l}\text { Total number of } \\
\text { complications }\end{array}$ & 56 & 26 & 38 & 1 & 121 \\
\hline Total women & $5.8(47)$ & $3.1(25)$ & $3.8(31)$ & $0.1(1)$ & 14.8 (119) \\
\hline
\end{tabular}


TABLE 7: Types of side effects / complications and severity reported by IUD clients 15 days post procedure ( $n=369)$

\begin{tabular}{|c|c|c|c|c|c|}
\hline \multirow{2}{*}{$\begin{array}{l}\text { Side effect } I \\
\text { complication }\end{array}$} & \multicolumn{4}{|c|}{ Level of severity \%(n) } & \multirow{2}{*}{$\begin{array}{l}\text { Total } \\
\%(n)\end{array}$} \\
\hline & $\begin{array}{l}\text { Very mild } \\
\quad \%(n)\end{array}$ & $\begin{array}{l}\text { Mild } \\
\%(n)\end{array}$ & $\begin{array}{l}\text { Moderate } \\
\%(n)\end{array}$ & $\begin{array}{l}\text { Severe } \\
\%(n)\end{array}$ & \\
\hline Pain & $5.7(21)$ & $2.4(9)$ & $2.4(9)$ & $0.8(3)$ & $11.4(42)$ \\
\hline Bleeding (menstrual) & $6.2(23)$ & $1.6(6)$ & $2.7(10)$ & $0.5(2)$ & $11.1(41)$ \\
\hline $\begin{array}{r}\text { Abdominal swelling / } \\
\text { pain }\end{array}$ & $3.5(13)$ & $0.5(2)$ & $0.5(2)$ & $0.3(1)$ & $4.9(18)$ \\
\hline Urinary problem & $0.5(2)$ & - & - & - & $0.5(2)$ \\
\hline IUD expulsion & & & & & $0.8(3)$ \\
\hline $\begin{array}{l}\text { Total complications } \\
\text { reported }\end{array}$ & 59 & 17 & 21 & 6 & 103 \\
\hline Total women & $12.0(45)$ & $4.0(15)$ & $4.0(15)$ & $1.0(4)$ & 21.4 (79) \\
\hline
\end{tabular}

and quick) surgical procedure, IUD insertion does not require a surgical procedure and is therefore considered less invasive. However, a higher proportion of IUD clients reported severe side effects, such as pain, bleeding and abdominal pain $(1 \%, n=4)$. Three women (1\%) reported that their IUD had come out by day 15 . Among the 79 women who reported any side effects, the majority experienced very mild or mild level of severity $(76 \%, n=60)$, and $24 \%(n=19)$ said their side effects were moderate or severe and required medical attention. In total 5\% $(n=19)$ out of 369 IUD clients reported a moderate or severe level of side effect which required medical attention.

\subsection{Follow up care and health seeking behaviour by day 15}

At the day 15 follow up interview women were also asked if they had sought medical care / advice or self-medicated in response to any of the side effects they had experienced. This question was asked to all women regardless of what level of severity of side effects they had experienced (which is also based on the level of intervention sought to help address these symptoms), in order to determine where these women went for medical care.

$5 \%$ of TL clients and $8 \%$ of IUD clients sought further medical care in addition to the follow up care advice and pain relief medication they were given as they left the outreach service. Among those TL and IUD clients who had reported any side effects and / or complications ( $n=119$ and $n=87$ respectively), one third of both TL clients $(34 \%, n=41)$ and IUD clients (32\%, $\mathrm{n}=28$ ) sought medical attention. The majority of women went to a government health centre for follow up care, followed by the MSI clinic for IUD clients and ANM and MSI IPC for TL clients. Although it is not known what medications were purchased, a smaller number of clients self-medicated by buying medicines from pharmacies / drug shops or visiting a private clinic (see Table 8).

8 In the level of severity definitions applied to measuring side effects a "mild" level could also include if a woman needed to self-medicate by buying pain relief from a pharmacy or drug store. Note that levels moderate and severe included seeking medical care from a health provider such as a nurse, private clinic or hospital. 
TABLE 8: Health seeking behaviour among women up to day 15 following TL and IUD procedure

\begin{tabular}{|c|c|c|}
\hline Health seeking behaviour & $\begin{array}{l}\text { Tubal ligation clients } \\
\qquad(n=803) \%(n)\end{array}$ & $\begin{array}{l}\text { IUD clients } \\
(n=369) \%(n)\end{array}$ \\
\hline Did not seek care & $94.8(762)$ & 93.0 (341) \\
\hline Self medicated & $0.8(7)$ & $1.3(5)$ \\
\hline Government HC / hospital & $2.8(23)$ & $3.7(14)$ \\
\hline Private clinic & $0.3(3)$ & $0.5(2)$ \\
\hline MSI clinic (static clinic) & 0 & $1.8(7)$ \\
\hline Others (ANM, MSI IPC) & $0.9(8)$ & 0 \\
\hline Total women sought care / advice & $5.1(41)$ & $7.5(28)$ \\
\hline
\end{tabular}

\section{Resumption of normal activities and work}

Among TL clients $94 \%$ ( $n=757)$ reported that they returned to work by the 15th day following their procedure. Being able to return to work for IUD clients was slightly more rapid than TL clients with 96\% $(n=359)$ resuming their normal activities by day 15 . Approximately $4 \%$ of women reported that they could not return to work for an average of 20 days.

\subsection{IUD discontinuation by day 90 post procedure}

During the follow up visit on day 90 IUD clients were asked about removal or expulsion of their IUD. Out of the 393 IUD clients who were interviewed at day 90, $92 \%$ were still using the method. Thirty two women (8\%) who attended the follow up interview on day 90 had discontinued their IUD (see Figure 5). Of these 32 women, $16 \%(n=5)$ reported that their IUD had been expelled and $84 \%(n=27)$ had had their IUD removed. Of the women whose IUD had come out, three of these women had reported the expulsion of their IUD at the time of the day 15 interview. The majority of women had their IUD removed at the health centre where the outreach services was carried out $(48 \%, n=13)$; others sought their removal from a private nurse or doctor $(41 \%, n=11)$ and a public health facility $(7 \%, n=2)$. One woman had removed the IUD by herself.
Women gave multiple reasons for wanting to have the IUD removed after 3 months. Out of the 27 women, the main reason given for removing their IUD was due to bleeding $(n=27)$, feeling weak ( $n=19)$, discomfort during intercourse $(n=6)$, husband objected $(n=4)$, fear the IUD may have moved inside the body $(n=3)$, fear will cause cancer $(n=3)$, and wanted to have another child $(n=1)$. There was also one report of IUD failure with one woman getting pregnant and consequently getting the device removed.

Among the women who decided to have their IUD removed or expelled $(n=32)$ and still did not want to get pregnant, only 15 women switched to an alternative method by day 90 . Methods that these women started to use were $T L(n=5)$, contraceptive pill $(n=7)$ and condom $(n=3)$.

\section{Side effects reported at day 90 post procedure}

Just over half of women $(54 \%, n=195)$ who still had their IUD when interviewed on day 90 said they did not experience any side effects or continuing problems associated with their IUD. Out of the women who did report side effects $(46 \%, n=166)$, the most frequently reported problem was menstrual bleeding problems and abdominal pain (see Figure 6). 
FIGURE 4: Flow chart showing IUD discontinuation by day 90 among IUD study cohort

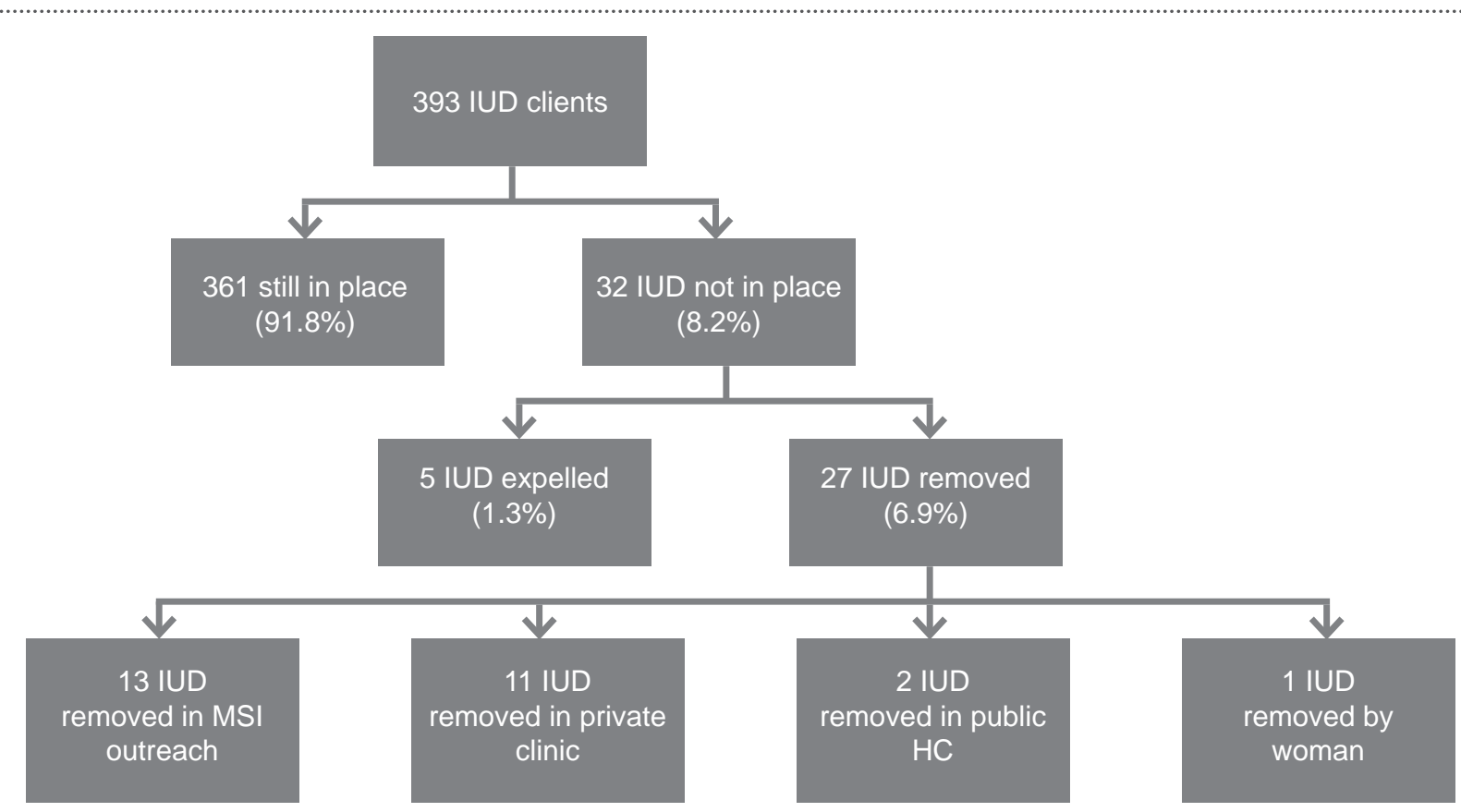

FIGURE 5: Self reporting of continued side effects of IUD at day 90 (\% of women) ( $n=393)$

Experienced no side effects

Excessive menstrual bleeding

Irregular menstrual cycle

in

Indigestion / discomfort in abdomen

Lower abdominal pain

$$
\text { in }
$$

Low backache

Prolonged menstrual bleeding

\section{g}

Excessive menstrual pain
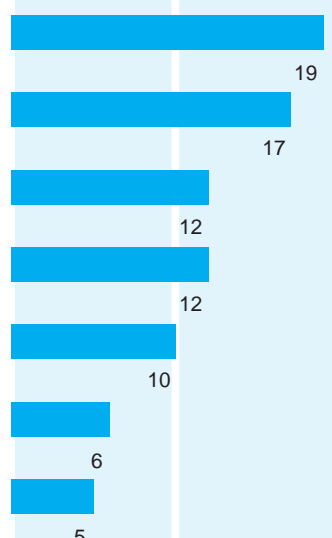


\subsection{Client Satisfaction}

Client satisfaction was recorded at day 15 and day 90 among IUD clients. Overall satisfaction with the outreach service was the same for both groups of women accessing TL and IUD services, with $98 \%$ of clients saying that the quality of services they received was either as expected or better than expected. 1.5\% $(n=6)$ of IUD clients were dissatisfied with the service due to the attitude of the doctor. Reasons cited by the small number of TL clients who were not satisfied with the service included lack of transport to the site, no provision of blankets and insufficient financial compensation for travel.

Women were also asked on the day of the procedure if they would recommend the method they received to a friend: $91 \%(n=792)$ of TL clients said they would recommend TL to a friend and $86 \%$ of IUD clients $(n=345)$. On day 90 the proportion of women who said they would recommend the IUD method to a friend decreased to $83 \%(n=326)$.

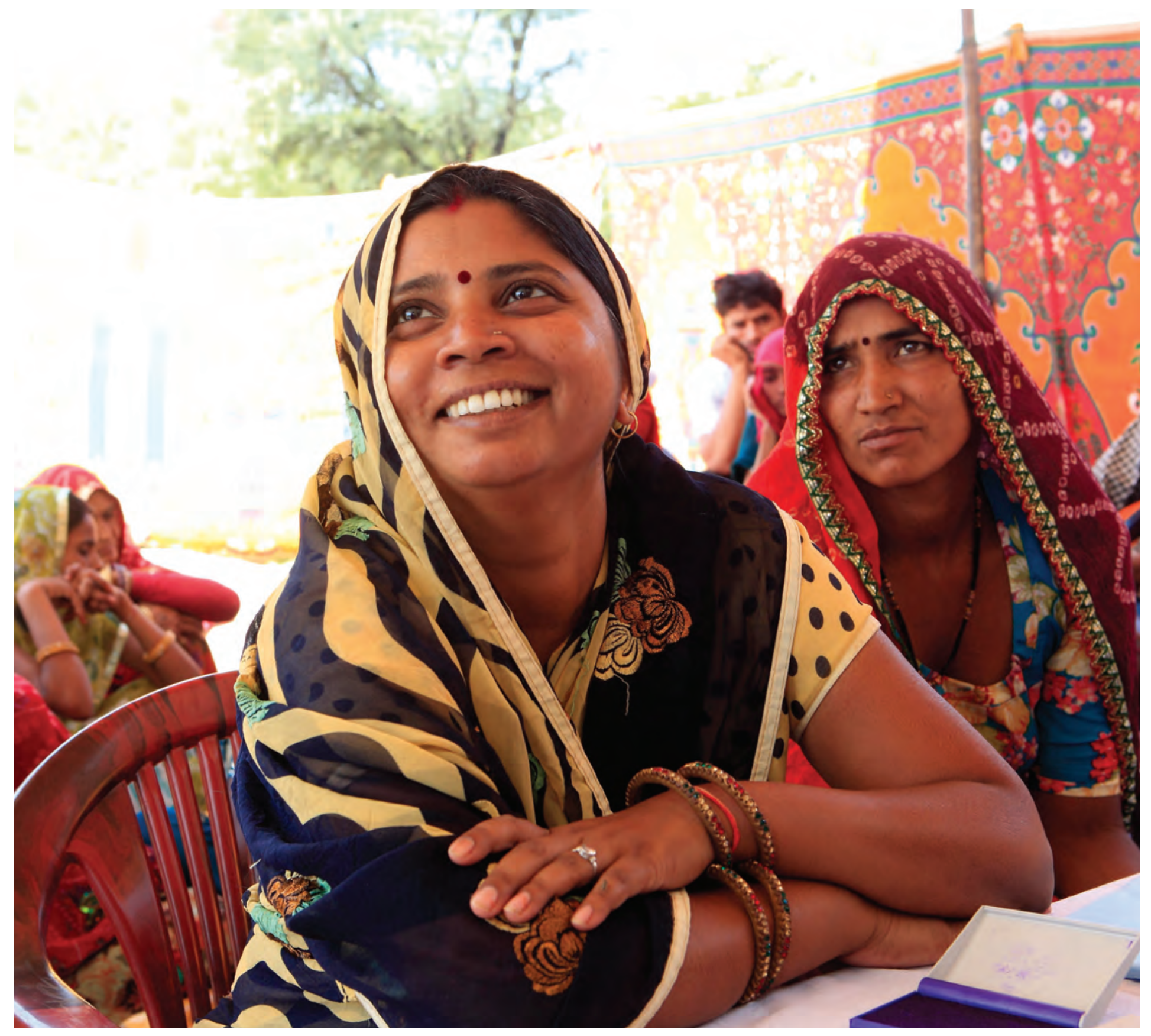




\section{Discussion and recommendations}

The study has shown that MS India provides TL and IUD contraceptive methods safely and effectively via mobile outreach in rural areas of Rajasthan, with low levels of minor complications and discontinuation, and a high level of user satisfaction. Furthermore the results indicate that MS India's outreach services are reaching under-served populations and addressing the contraceptive unmet needs of poor and marginalised men and women in the state of Rajasthan, with the majority of TL and IUD clients living in rural areas (81\% and $72 \%$ ), having never attended school (53\% and $30 \%$ ), and belonging to disadvantaged groups (classified in India as Other Backward Classes; 53\% and 62\%). Although the findings showed that the majority of TL and IUD clients came from households with a high SLI (TL 44\% and IUD 61\%), the proportion of TL clients who have a low SLI (25\%) is comparable to the proportion of the population in Rajasthan who are of low income level households $(26 \%)^{9}$.[12] A higher proportion of TL clients than IUD clients are from households with a low or medium SLI (56\% and $39 \%)$. Prior to receiving LARC or PM via outreach services, $73 \%$ of TL clients and $54 \%$ of IUD clients were not using any form of contraception, and $63 \%$ of TL clients and $41 \%$ of IUD clients had never used contraception before. The study did not collect data on what method those using contraception had been using prior to receiving their LARC or PM method. The majority of women choosing to have a TL had two to three children (76\%) and the majority of IUD clients had one to two children (59\%) demonstrating the importance of expanding contraceptive choices for women in hard to reach areas who wish to space as well as limit the number their number of births.

Women learn about contraception mainly from the government ANM, spouses or community health workers. While a majority of women were able to name family planning methods, the study did not measure their level of understanding of these different methods, which is a limitation. Knowledge of IUDs was low, and the fact that $22 \%$ of IUD discontinuations were related to myths and misconceptions around IUDs (for example causing cancer or moving in the body) demonstrates the need to provide more comprehensive information about this method.

The main decision women gave for visiting MS India's outreach sites were because these services were free and medical personnel were available. A large proportion of women also reported the influential role of their husbands or other family member in their decision to adopt a TL or IUD. This highlights the important role these people play in women's contraceptive decision making and the need for outreach services to fully incorporate them in educational activities to ensure both men and women are fully informed about all contraceptive methods available.

\section{Incidence of side effects and complications}

The reported incidence of side effects and complications in this study are not dissimilar to those documented in the published literature. One third of all women who had an IUD fitted said they experienced some side effects following their procedure (32\%, $n=94$ ). Side effects experienced by women following insertion of an IUD were generally mild to moderate with less than $1 \%(n=2)$ reporting severe pain. At day 15 , one in four women who had an IUD fitted (23\%, $\mathrm{n}=87$ ) reported still experiencing at least one type of side effect, with $1 \%(n=4)$ reporting a severe complication relating to pain, bleeding and abdominal pain / swelling. According to the literature, complications during IUD insertion, such as cervical laceration or uterine perforation are rare. [5, 15] However, most women experience some minor side effects such as changes in menstrual cycle and pain. In a similar study conducted in 2010 by MSI among clients receiving LARCs and PMs in outreach settings in Uganda, it found 3\% of IUD clients experience severe side effects immediately after insertion and 1.9\% experiencing minor complications at day 15. [6] In another study carried out in 2009 in a clinical setting, $9 \%$ of the 1,947 participants reported serious pain in the first nine weeks of IUD use. [19] In this study, even though the cases were not verified by a medical practitioner, they highlight important areas to be addressed.

One third of women who adopted a TL also experienced side effects following their procedure (35\%, $n=308)$ with the majority being described as mild to moderate, and $2 \%(n=18)$ reporting severe pain. At day 15 following the procedure, $15 \%(n=119)$ of TL

9 As documented in the DLHS3, for 2007-2008 survey the SLI index for the state of Rajasthan was low 25.5\%; medium $29.5 \%$; and high $28.8 \%$. 
clients reported still experiencing at least one type of side effect. Studies have shown that TL is generally associated with low levels of minor complications (1-4\%), such as wound infections and small haematoma which can be resolved easily and rapidly. [10] For example, in the same study cited above among MSI clients receiving LARCs and PMs in outreach settings in Uganda, it found 1\% of TL clients experienced minor complications at the day 15 follow up interview and a further $6 \%$ experienced frequent pain.[6] In this study, $13 \%(n=118)$ of TL clients reported very mild-moderate complications at day 15 and only $0.1 \%(n=1)$ reported severe complications related to bleeding at the site of the wound.

\section{IUD discontinuation}

All IUD insertions were interval insertions. In the current study a total of $8 \%(n=32)$ of IUD clients had discontinued their IUD at day 90 . Of these women $1 \%$ $(n=5)$ reported their IUD had been expelled. This is comparable with other published studies that have shown the proportion of women experiencing expulsion of the IUD ranging from less than $1 \%$ to $10 \%$ annually, although it is reported that most of these occur within the first three months. $[5,11]$ There is evidence to suggest that correct insertion technique can reduce the rate of expulsion. [5]

A relatively small proportion, $7 \%(n=27)$ of women decided to have their IUDs removed by day 90 . However this removal rate is slightly higher than that reported in other studies conducted in similar nonclinical settings: the MSI outreach LARCs and PM study in Uganda found an IUD removal rate of $6 \%$ at 90 days; [6] and the results of a retrospective multicountry study assessing discontinuation rates of IUDs revealed $3 \%$ and $5 \%$ of IUD clients had IUDs removed within three months in Pakistan and Myanmar respectively.[9] In this study, women who had their IUD removed said they did so due to bleeding ( $n=27)$, feeling weak $(n=19)$ or discomfort and pain $(n=6)$. Among the women who discontinued their IUD only half of these women switched to an alternative method of contraception which highlights the need to have mechanisms in place for appropriate follow up and counselling for alternative methods. One case of method failure was reported; the woman became pregnant with her IUD in situ and therefore had it removed. This case was recorded in the 90 day follow up.

It may be possible to reduce discontinuation by strengthening counselling so that women are aware of expected side effects and are informed that these should dissipate over time. Expected side effects should be clearly distinguished from those that are not expected, are serious and require women to seek medical help. Qualitative interviews among the IPC also revealed that the prevailing myths and misconceptions about the safety and efficacy of the IUD (and vasectomy) - such as the IUD causing cancer, or that it can float around the body, and that vasectomy is unsafe for a man - are a barrier for men and women to adopt these methods. Campaigns could be developed to dispel these myths and increase uptake and continuation of this effective method.

Despite MS India's standard follow up care process, including visits by the government ANM and provision of pain relief and other medication, one third of TL and IUD clients who reported side effects (TL $n=38$; IUD $\mathrm{n}=28$ ) still required post procedural care and resorted to seeking further medical advice or self-medicated to treat their on-going side effects. Post procedure follow up care services may need to be reviewed to ensure that the provision of personnel and supplies are consistently available in all outreach areas.

High client satisfaction ratings among TL and IUD methods and a willingness of women to recommend the outreach services to others are encouraging. Satisfaction among users of IUD declined slightly from day 15 to day 90 which may indicate a need for more counselling regarding the duration of some expected side effects.

\section{Limitations of the study}

This service evaluation has several limitations.

1. The SLI used in this study is based on different measures of poverty from the tool used in the MSI Exit Interview Survey (used across the MSI partnership). While this provides a sufficient measure in the India context it does not provide a reliable comparison with other MSI programmes or other poverty data routinely collected by MS India. The data in this survey shows MS India reaching 
women of more high income level households which is at odds with MSI data that demonstrates that a higher proportion of clients are poor. This may be more a reflection of the different definitions and methods used to determine "poor" than to suggest the clients included in this study are of a different socio-economic status than the usual profile of MS India's outreach programme in Rajasthan.

2. Types of side effects and severity of side effects as well as IUD expulsion were self-reported and not verified by medical personnel, which may have resulted in some over reporting. However, these self-reports reflect the actual experiences of women and should be not be underestimated in efforts to improve counselling and awareness raising activities around long acting reversible and permanent contraceptive methods.

3. The follow up period of 15 days and then 90 days for IUD was relatively short, and therefore the study does not provide a good reflection of longer term side effects, such as changes in menstrual bleeding which may emerge over time, or of discontinuation behaviours over a longer term. It would also have been informative to have recorded the actual number of days it took both TL and IUD clients to recover and resume their normal activities to further verify the severity of side effects and impact on women's lives.

4. Due to the small sample size (related to the duration and available resources for the study) it was not possible to further analyse the characteristics of women who did experience complications and discontinued their IUD. As with other prospective studies measuring post procedure outcomes, without strict medical verification and control, it is difficult to differentiate side effects / complications as a result of the procedure from symptoms relating to the clients' conditions or living environment that may exacerbate complications, such as post procedure wound care. Therefore for this study it is difficult to determine the exact cause of the (small) number of complications.

5. Clients were asked about satisfaction with the service in general but not specifically about different aspects of the service such as consultation, counselling, procedure, pain management and recovery, nor about satisfaction with the particular method adopted.

\subsection{Conclusions}

The study demonstrates the safety and effectiveness of providing TL and IUDs through MS India's mobile outreach services in the state of Rajasthan, and the importance of continuing to use this service model to increase access and choices for poor men and women living in rural areas. By providing TLs and IUDs in underserved, hard to reach areas, MS India significantly contributes to the improvement of Rajasthan's overall sexual and reproductive health service provision. This service evaluation has also helped to identify areas to strengthen and improve MS India's outreach model, as well as to be considered by other mobile outreach programmes.

\subsection{Recommendations}

It is recommended that the MS India program consider the following:

- Strengthen counselling to increase client awareness of expected side effects of LARCs and PMs to help reassure new-users of these symptoms, how to manage them and to emphasise that these will dissipate in time. Counselling also needs to clearly distinguish between expected and unexpected side effects of LARCS and PMS and to more clearly outline what to do if complications arise. This can help to reduce women seeking unnecessary medical care and to ensure women have sufficient information on likelihood and duration of side effects to make an informed decision to continue with or discontinue their IUD.

- To address some of the factors cited by women who had their IUD removed, as well as to encourage other women to adopt this effective, reversible method of family planning, the program could benefit from conducting further qualitative research to explore the myths surrounding longacting reversible methods and develop campaigns to try to dispel misconceptions and increase uptake and continuation. 
- All awareness raising activities for outreach services and counselling about LARCs and PMs need to acknowledge and involve the role of the husband and other key family members who influence women's decision making about family planning. Whilst informed consent is that of the woman's it is important that close family members are well informed about all contraceptive methods available as well as post-procedure care.

- MS India should continue to monitor the clinical outcomes of TL and IUD clients attending outreach services including monitoring contraceptive compliance so that women who are considering or have already had the IUD removed due to side effects, and who still have an unmet need, receive additional counselling to help them deal with their symptoms better or alternatively are offered a different method of contraception.

- Continue to provide supportive clinical supervision to outreach teams and review post-procedure counselling and care. In particular, ensure clients are provided adequate analgesic medication following the procedure and understand how to check if their IUD is in place.

- Continue to establish and maintain important networks with other health providers who are able to address complications and side effects effectively.

- As a reproductive health right for all women MS India should continue to advocate for the registration of implants in India and the expansion of access to contraceptive injectables in the public sector, to enable women to have more choice of family planning methods.

\subsection{Evidence to Action: Marie Stopes India response to recommendations}

Following the evaluation of MS India's outreach services which were conducted by the Population Council in India in 2012, MS India have incorporated a number of the recommendations into routine programming in order to improve access and quality of the outreach services:
- In order to strengthen all aspects of counselling on all methods, an intensive three day training for all the field level staff was facilitated and concluded. In addition, the recommendation to increase awareness among clients of expected side effects of LARCs and PMs has been taken on board, with additional focus on this area during provider training. To improve post procedure counselling and follow up, a new system has been instituted that ensures a 24 hour follow-up. The nurses in the clinical outreach teams have been provided with a mobile handset to ensure that the calls are made en-route to the outreach site the following day. This follow-up ensures that the team is better connected with the clients, and helps them understand any issues that they may have with the method provided. Post procedure counselling is also being strengthened through a mobile phone SMS-based project, currently being trialled with SIFPO-MSI funding, which gives consistent and systematic reminders to the nurses to improve post procedure care and counselling.

- MSI has evolved its approach to outreach to include the sub-health centre level, so that IUD services are now available at the sub centre level. Readier access to IUD services has increased uptake.

- All doctors and the nurses have also been trained on IUD insertions to ensure quality services.

- Internal audits to assess quality of services provided at all outreach sites have now been systematically rolled out. Every team is reviewed once a quarter by the technical quality team.

MS India continues to work to ensure that the safety and effectiveness of its outreach programme is expanding choice and access to contraception for poor men and women living in rural and hard to reach areas of India. 


\section{References}

1. Kulier, R., et al., Effectiveness and acceptability of progestogens in combined oral contraceptives - a systematic review. Reprod Health, 2004. 1(1): p. 1.

2. IIPS, M., Int., National Family Health Survey (NFHS-3), 2005-06 India: Key Findings. 2008, International Institute for Population Sciences (IIPS): Mumbai.

3. WHO, Medical eligibility criteria for contraceptive use. 2004, World Health Organisation: Geneva.

4. MoHFW, Family welfare statistics in India. . 2011, Statistics Division, Ministry of Health and Family Welfare, Government of India.

5. Mishell, D.R., Intrauterine devices: mechanisms of action, safety, and efficacy. Contraception, 1998. 58(3): p. 45S-53S.

6. Reiss, K., Natayi, L., Odong, J. \& Ngo, T.D. , Providing long acting and permanent contraceptive through outreach in rural Uganda. . 2012, Marie Stopes International: London, UK.

7. Ruminjo, J.K. and P.F. Lynam, A fifteen-year review of female sterilization by minilaparotomy under local anesthesia in Kenya. Contraception, 1997. 55(4): p. 249-60.

8. Chipeta-Khonje, A., Ghosh, S., Corby, N. and Ngo, T. , Marie Stopes tubal ligations: a rapid assessment of complication rates and client satisfaction in Malawi, 2008. 2008, Marie Stopes International: London.

9. Eva, G., \& Ngo, T., MSI Mobile Outreach Services: Retrospective evaluations from Ethiopia, Myanmar, Pakistan, Sierra Leone and Vietnam. 2010, Marie Stopes International: London.

10. WHO, Female Sterilization: What health workers need to know. 1999, World Health Organization: Geneva.

11. Kulier, R., O’Brien, P., Helmerhorst, F., UsherPatel, M., D'Arcangues, C., Copper containing, framed intra-uterine devices for contraception (Review). . 2008, The Cochrane Library 2008.

12. IIPS, District Level Household Survey (DHLS-3), 2007-2008. 2008, International Institute for Population Sciences (IIPS): Mumbai, India.
13. Santhya, K.G., Changing family planning scenario in India: An overview of recent evidence. 2003, Population Council: New Delhi, India.

14. Unknown. Meeting the unmet need for family planning in India. 2013 [27 February]; Available from: http://southasia.oneworld.net/news/meetingthe-unmet-need-for-family-planning-in-india.

15. WHO, Intrauterine devices: Technical and managerial guidelines for services, . 1997, World Health Organization: Geneva.

16. United Nations, D.o.E.a.S.A., Population Division, Levels and trends of contraceptive use as assessed in 2002. 2006: New York.

17. Aruldas, K., Khan, M. E., Ahmad, J., \& Dixit, A. , Evaluation of female sterilization within Marie Stopes International mobile outreach services in Rajasthan, India. 2013, Population Council.: New Delhi, India.

18. Aruldas, K., Khan, M. E., Ahmad, J., \& Dixit, A., Evaluation of mobile outreach IUCD services provided by Marie Stopes International in Rajasthan, India. 2013, Population Council.: New Delhi, India.

19. Hubacher, D., Chen, P-L., Park, S., Side effects from the copper IUD: do they decrease over time? Contraception, 2009. 79: p. 356-62.

20. K.S. Babu, Townsend, J.W., RamaRao, S. 1995. Evaluation of mobile education and service units in increasing accessibility and acceptability of family planning methods. Available at: http://pdf. usaid.gov/pdf_docs/PNACC052.pdf

21. Marie Stopes International (2009) Clinical Quality Standards, One World One Standard. London: MSI 
Marie Stopes International

1 Conway Street

Fitzroy Square

London W1T 6LP

$\mathrm{t}:+44(0) 2076366200$

f: +44 (0)20 70342369

e: info@mariestopes.org

w: www.mariestopes.org

Registered charity number: 265543

Company number: 1102208
Population Council

One Dag Hammarskjold Plaza

9th Floor

New York, NY 10017

USA

t: +1 2123390500 